\title{
Variabilidad estacional de la distribución y abundancia de larvas de langostino colorado en la zona norte de Chile (Decapoda, Anomura, Galatheidae)
}

\author{
Jorge Rivera \& Edgardo Santander \\ Departamento de Ciencias del Mar, Universidad Arturo Prat \\ Casilla 121, Iquique, Chile
}

\begin{abstract}
RESUMEN. Se analiza la distribución y abundancia de los estadios larvales de langostino colorado Pleuroncodes monodon en la zona norte de Chile, capturados entre 0 y $100 \mathrm{~m}$, en cuatro cruceros oceanográficos efectuados en

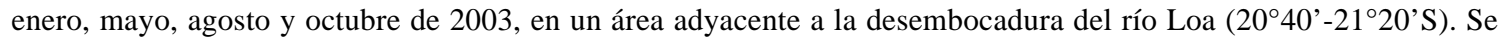
realizó un total de 20 estaciones oceanográficas y las muestras zooplanctónicas se obtuvieron mediante redes Bongo desde la superficie hasta $100 \mathrm{~m}$ de profundidad, registrándose además datos de temperatura, oxígeno disuelto y densidad en cada estación. Los máximos de abundancia se registraron en enero y agosto, y el mínimo en mayo. En sentido norte-sur, las mayores abundancias se encontraron en las secciones situadas en el sector norte. No obstante, en octubre se observó una distribución de abundancia más homogénea. En sentido costa-océano se determinó un patrón diferenciado, con una preferencia oceánica de los estadios zoea III-V y megalopa. Además, se observó una tendencia estacional de los focos de mayor abundancia larvaria asociados a las estaciones con temperaturas medias de $14,0-16,0^{\circ} \mathrm{C}$ y a concentraciones medias de oxígeno disuelto de $1,58-4,55 \mathrm{~mL} \cdot \mathrm{L}^{-1}$.
\end{abstract}

Palabras clave: Pleuroncodes monodon, Galatheidae, estadios larvales, variabilidad espacio-temporal, abundancia, norte de Chile.

\section{Seasonal variability in the distribution and abundance of larvae of the red squat lobster (Decapoda, Anomura, Galatheidae) from Northern Chile}

\begin{abstract}
An analysis was made of the distribution and abundance of red squat lobster (Pleuroncodes monodon)

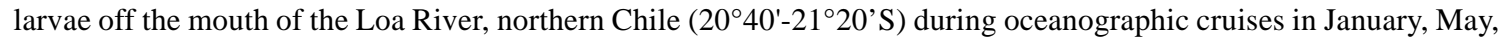
August and October 2003. A total of 20 oceanographic stations were occupied, with zooplankton samples taken using Bongo nets towed from the surface to $100 \mathrm{~m}$ depth. Other data obtained at each station included temperature, dissolved oxygen, and seawater density. Maxima of abundance of these larvae were recorded in January and August, with a minimum in May. On N-S tracks the highest abundances were recorded in the northern sector. A more homogeneous distribution of abundance was observed in October. On E-W tracks a differentiated pattern was observed, where zoea stages III-V and megalopae occurred in the oceanic (westward) samples. Also, a seasonal tendency was noted, with foci of larval abundance associated with stations having average temperatures of $14.0-16.0^{\circ} \mathrm{C}$ and dissolved oxygen concentrations of $1.58-4.55 \mathrm{~mL} \cdot \mathrm{L}^{-1}$.
\end{abstract}

Keywords: Pleuroncodes monodon, Galatheidae, larval stages, space-temporal variability, abundance, northern Chile. 


\section{INTRODUCCIÓN}

La distribución del langostino colorado Pleuroncodes monodon (H. Milne Edwards, 1837), se extiende desde la isla Lobos de Afuera, Perú hasta Ancud, Chile (Haig, 1955; Retamal, 1981). Sin embargo, su área de extracción pesquera en aguas chilenas se encuentra restringida entre Coquimbo $\left(30^{\circ} \mathrm{S}\right) \mathrm{y}$ Talcahuano ( $\left.37^{\circ} \mathrm{S}\right)$ (Gallardo et al., 1993; Palma, 1994). En estas zonas, el langostino colorado vive entre 50 y $350 \mathrm{~m}$ de profundidad, siendo una especie de hábitos bentodemersales y su distribución batimétrica varía en función de su comportamiento reproductivo (Bustos et al., 1982; Palma \& Arana, 1997).

En cambio, en la zona norte de Chile se ha señalado que la distribución del langostino colorado sería más bien de tipo pelágica y se distribuye en aguas relativamente superficiales entre $0-100 \mathrm{~m}$ de profundidad (Gutiérrez \& Zúñiga, 1977). Con relación a esto, el langostino colorado del norte es el crustáceo decápodo que más se ha asociado a la pesca de la anchoveta durante los últimos años y debido a la falta de hallazgos de ejemplares bentónicos en esta zona, se ha generado una controversia sobre la verdadera identidad de esta especie, llegando a describirla, posiblemente como una subespecie enana de hábitos pelágicos (P. monodon pelagicus) (P. Báez \& G. Guzmán, com. pers.).

La masiva ocurrencia de langostino colorado del norte en la columna de agua, implica la presencia de larvas en el plancton, no existiendo a la fecha información sobre la ecología planctónica de esta especie en la zona norte. Por otra parte, son pocos los estudios realizados sobre la ecología larval de $P$. monodon, en el resto del país, destacando los trabajos de Palma (1994) en aguas frente la costa de Concepción, Palma $(1976,1980)$ sobre las larvas de decápodos frente la costa de Valparaíso y un manuscrito no publicado de Mujica \& Bahamonde (1990), sobre larvas de Galatheidae frente a Talcahuano.

El objetivo del presente trabajo es analizar la distribución espacial y estacional de las larvas de langostino colorado en el norte de Chile y su relación con las condiciones ambientales durante el 2003. La información analizada proviene del Programa de Investigación en Oceanografía Pesquera (PROPNOR), realizado frente a la zona adyacente de la desembocadura del río Loa, como parte del convenio de cooperación entre la Universidad Arturo Prat y la empresa pesquera Corpesca S.A., el cual comprende una serie de investigaciones multidisciplinarias destinadas a aportar nuevos antecedentes sobre la ecología de especies de interés pesquero en el norte de Chile.

\section{MATERIALES Y MÉTODOS}

Entre enero y octubre de 2003 se efectuaron cuatro cruceros oceanográficos, frente a la desembocadu-

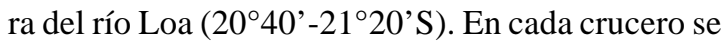
efectuaron cinco transectas perpendiculares a la costa, en las cuales se distribuyeron 20 estaciones oceanográficas ubicadas a 1, 5, 10 y $15 \mathrm{mn}$ de la costa (Fig. 1). Los cruceros se efectuaron con la goleta de cerco PAM "El Roble" de la compañía pesquera Corpesca S.A., entre el 21 y 22 de enero (verano), 2 y 3 de mayo (otoño), 9 y 10 de agosto (invierno) y 16 y 17 de octubre (primavera). En el crucero de mayo se realizaron solo 13 estaciones con recolección de material biológico, debido a la pérdida del sistema de redes.

El muestreo de zooplancton se efectuó mediante pescas dobles oblicuas realizadas entre la superficie y una profundidad máxima de 100 m, con una

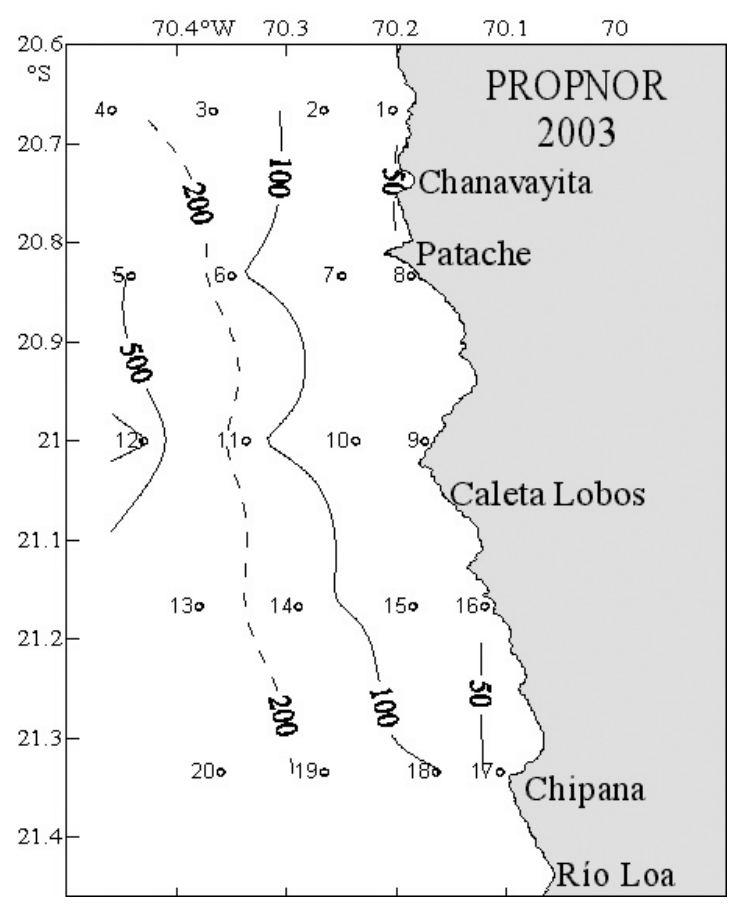

Figura 1. Ubicación de las estaciones oceanográficas en el área de estudio.

Figure 1. Location of the oceanographic stations in the study area. 
red Bongo de $60 \mathrm{~cm}$ de diámetro, con mallas de 300 y $500 \mu \mathrm{m}$, provista de un flujómetro Hydrobios para la estimación del volumen de agua filtrada. Las muestras se fijaron en una solución de agua de mar con formalina (5\%) neutralizada con bórax.

Las muestras obtenidas con malla de 500 $\mu \mathrm{m}$ se revisaron y subdividieron con un separador Folsom hasta 1/4, 1/8 y en algunos casos, hasta $1 / 16$. De estas submuestras se separaron y contabilizaron todas las larvas de crustáceos decápodos y en particular las de langostino colorado, que se preservaron en etanol (96\%) para su análisis en laboratorio. La identificación taxonómica se verificó mediante las descripciones larvales de Pleuroncodes monodon (Fagetti \& Campodonico, 1971; Bustos et al. 1982), P. planipes (Boyd, 1960; Boyd \& Johnson, 1963) y otras descripciones de larvas de distintos géneros de galateidos (Rayner, 1935; Gore, 1979; Christiansen \& Anger, 1990; Seridji, 1995).

La distribución espacio-temporal de los distintos estadios larvales se expresó en número de individuos por $10 \mathrm{~m}^{-2}$ de superficie de agua de mar y su abundancia se representó gráficamente en escala métrica $\log ^{2}$, que permite determinar clases de abundancias equidistantes (Frontier, 1981; Palma, 1994).

Los datos oceanográficos se registraron mediante un CTD (SeaBird-19) y botellas Niskin, determinando la temperatura, densidad y concentración de oxígeno disuelto en la columna de agua. Para determinar la relación entre la abundancia relativa de larvas y las variables ambientales, se utilizó el promedio ponderado de estas variables hasta $\operatorname{los} 25 \mathrm{~m}$, profundidad aproximada que alcanzó la base de la termoclina, oxiclina y picnoclina en el área de estudio, mediante la siguiente fórmula (Rosales, 1992; Ulloa \& Palma, 1998):

$$
X_{i j}=\frac{\sum_{k=1} Z_{j k} \cdot C_{i j k}}{\sum Z_{j k}}
$$

donde:
$X_{i j} \quad$ : promedio ponderado del i-ésimo parámetro de la j-ésima estación.

$Z_{j k} \quad$ : k-ésima profundidad de la j-ésima estación.

$C_{i j k} \quad: \quad$ valor del i-ésimo parámetro en la j-ésima estación a la k-ésima profundidad.

Finalmente, para determinar el grado de asociación entre los distintos estadios larvales, se utilizó el índice de disimilitud de Bray-Curtis (Brower et al., 1997), resumiendo los resultados en dendrogramas para su posterior interpretación.

\section{RESULTADOS}

\section{Variación estacional}

La variación estacional de los estadios tempranos, permitió observar que las zoeas I y II comprendieron la fracción más abundante con el 56 y $26 \%$ respectivamente. Las zoeas III, IV y megalopa no superaron el $6 \%$ cada una de ellas, siendo la zoea $\mathrm{V}$ la de menor representatividad (1\%) en los períodos analizados (Fig. 2).

En enero se determinó una media de 1.378 ind. $\cdot 10 \mathrm{~m}^{-2}$, donde la zoea I fue la más abundante con 21.298 ind. $\cdot 10 \mathrm{~m}^{-2}$, seguida de la megalopa con 2.720 ind. $\cdot 10 \mathrm{~m}^{-2}$. El resto de los estadios estuvo poco representado con abundancias que no superaron los 1.777 ind. $\cdot 10 \mathrm{~m}^{-2}$. En mayo la abundancia disminuyó drásticamente en todos los estadios larvales, con una media de 54 ind. $10 \mathrm{~m}^{-2}$, donde las megalopas fueron las más abundantes con 340 ind. $10 \mathrm{~m}^{-2}$.

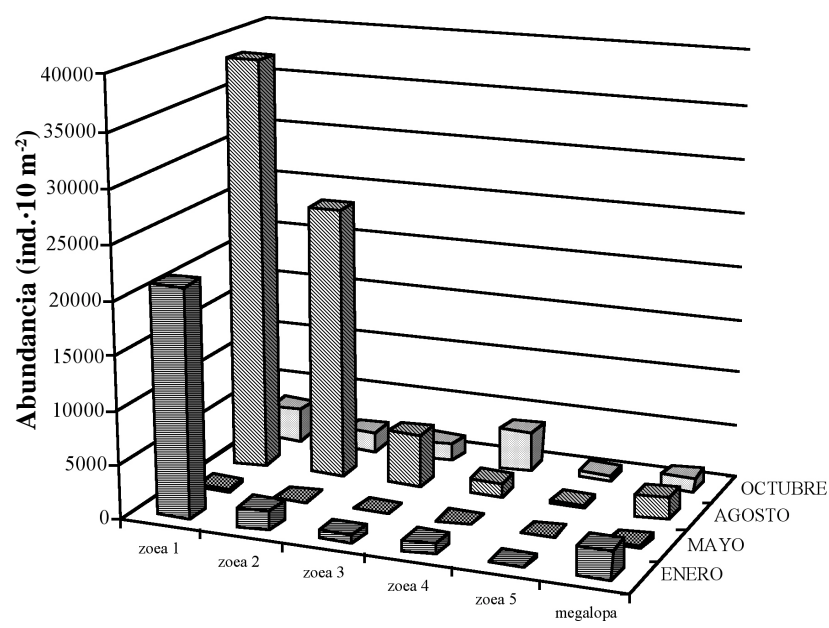

Figura 2. Abundancia estacional de cada estadio larval de langostino colorado en el norte de Chile.

Figure 2. Seasonal abundance of each larval stage of the red squat lobster from northern Chile. 
En agosto se estimó el máximo de abundancia, con valores medios de 3.646 ind. $10 \mathrm{~m}^{-2}$, donde las zoeas I y II fueron las más abundantes, con 38.600 y 25.276 ind. $.10 \mathrm{~m}^{-2}$ respectivamente. Sin embargo, el notable incremento en invierno, se redujo a una media de 924 ind. $10 \mathrm{~m}^{-2}$ en octubre (primavera), cuando se observó una mayor similitud en la abundancia de los distintos estadios larvales, destacando la zoea IV con 3.767 ind. $10 \mathrm{~m}^{-2}$, seguida de la zoea I con 3.224 ind. $10 \mathrm{~m}^{-2}$ (Fig. 2).

\section{Variación norte-sur}

En el sentido norte-sur se observó una mayor concentración de larvas en el sector norte durante el periodo de estudio (Fig. 3). En casi todos los cruceros los estadios tempranos, zoeas I y II estuvieron distribuidos homogéneamente, con una mayor abundancia en las secciones norte y central, salvo en octubre donde se distribuyeron preferentemente en la sección central (Figs. 4 y 5).

En las zoeas III a V, no se observó una tendencia norte-sur, estando presente en todas las secciones pero en baja abundancia relativa (Figs. 6 y 7). Sin embargo, se observó una mayor abundancia en las secciones norte, principalmente en enero y agosto donde ocurrieron las mayores abundancias totales. El estado de megalopa fue más abundante en las secciones norte y central, registrándose su presencia durante los cuatro cruceros analizados, aunque con mayor abundancia en enero y agosto (Fig. 8).

\section{Variación costa-océano}

La distribución espacial de larvas de P. monodon en sentido costa-océano, no mostró ningún patrón diferenciado en cuanto a su abundancia total (Fig. 3). No obstante, se constató una distribución diferenciada entre los distintos estadios larvales.

En las zoeas tempranas I y II se observó una distribución preferentemente en la banda costera hasta las $10 \mathrm{mn}$, disminuyendo su abundancia hacia las $15 \mathrm{mn}$. Sin embargo, en agosto este patrón no se observó, encontrándose focos de abundancias en casi todas las estaciones, tanto costeras como oceánicas (Figs. 4 y 5). La zoea III, mostró una distribución amplia en sentido costa-océano, con tendencia a alejarse de las aguas costeras, en enero y agosto principalmente (Fig. 6). En cambio, las zoeas avanzadas IV y V mostraron un claro alejamiento desde la zona costera durante los cuatro períodos analizados, distribuyéndose preferentemente entre las 10 y 15 mn (Figs. 7 y 8). Las megalopas presentaron una distribución intermedia, con sus mayores abundancias entre las 5 y $10 \mathrm{mn}$ principalmente (Fig. 9).

\section{Condiciones hidrográficas}

En el estrato de 0-25 m se observó una banda costera relativamente fría durante los cuatro cruceros, con temperaturas de $15,4-17^{\circ} \mathrm{C}$ en enero, $14,5-15,6^{\circ} \mathrm{C}$ en mayo, $13,7-15,2^{\circ} \mathrm{C}$ en agosto y $13,9-15,6^{\circ} \mathrm{C}$ en octubre. Además, se observaron gradientes térmicos entre la costa y la zona más oceánica durante los cuatro periodos, siendo éstos más marcados en enero y octubre. En agosto se determinó una relativa homogeneidad térmica en la sección norte (Fig. 10). En los cuatro periodos del año se advirtió un patrón característico en la sección sur, frente a punta Chipana, donde se registraron los mayores valores de temperatura en las estaciones más oceánicas, con fuertes gradientes térmicos horizontales en las secciones sur (Fig. 10).

La distribución de oxígeno disuelto mostró una mayor variabilidad estacional y espacial, con mínimos en enero $\left(1,05 \mathrm{~mL} \cdot \mathrm{L}^{-1}\right)$ y máximos en agosto $\left(6,25 \mathrm{~mL} \cdot \mathrm{L}^{-1}\right)$. La distribución en sentido costa-océano fue relativamente concordante con la estructura térmica, observándose aguas poco oxigenadas en las estaciones costeras, salvo en agosto en que se detectaron aguas bien oxigenadas en casi toda el área (Fig. 11). Hacia el sector oceánico se observaron aguas bien oxigenadas, con valores máximos en agosto. En sentido norte-sur se registró un aumento de los promedios ponderados de oxígeno disuelto hacia el sector sur y lejos de la costa (Fig. 11).

El campo de la densidad (expresado en unidades sigma-t) presentó una marcada estacionalidad en los primeros $25 \mathrm{~m}$ de profundidad. Las menores densidades se observaron en enero ( $<25,3$ sigma-t) y las mayores en agosto y octubre, 26,45 y 26,67 sigma-t respectivamente. Al igual que lo descrito para la temperatura, se determinaron gradientes o frentes en los cuatro cruceros, estando más alejados de la costa en enero y agosto, que en mayo y octubre en que fueron más costeros y presentes principalmente en las secciones sur (Fig. 12). En contraste, en agosto y octubre se observó una relativa homogeneidad en el sector norte (Fig. 12). 

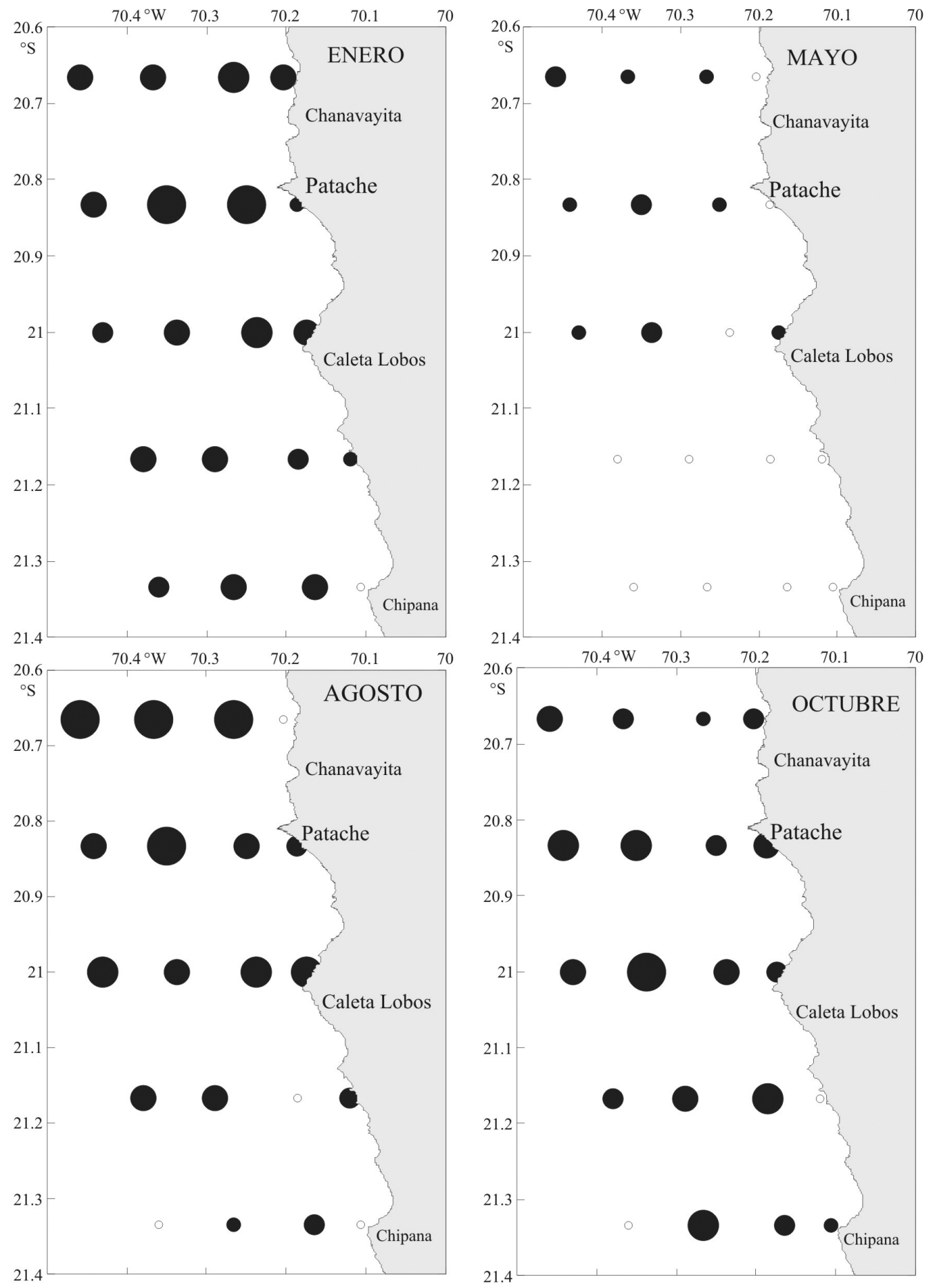

${ }^{\circ} 0 \quad \bullet 1$ a 64

64 a 365

365 a 1.400

1.400 a 4.200

4.200 a 11.500

Figura 3. Distribución temporal de larvas de langostino colorado en el norte de Chile (ind. $10 \mathbf{~ m}^{-2}$ ).

Figure 3. Temporal distribution of the red squat lobster larvae from northern Chile (ind. $\cdot 10 \mathbf{~ m}^{-2}$ ). 

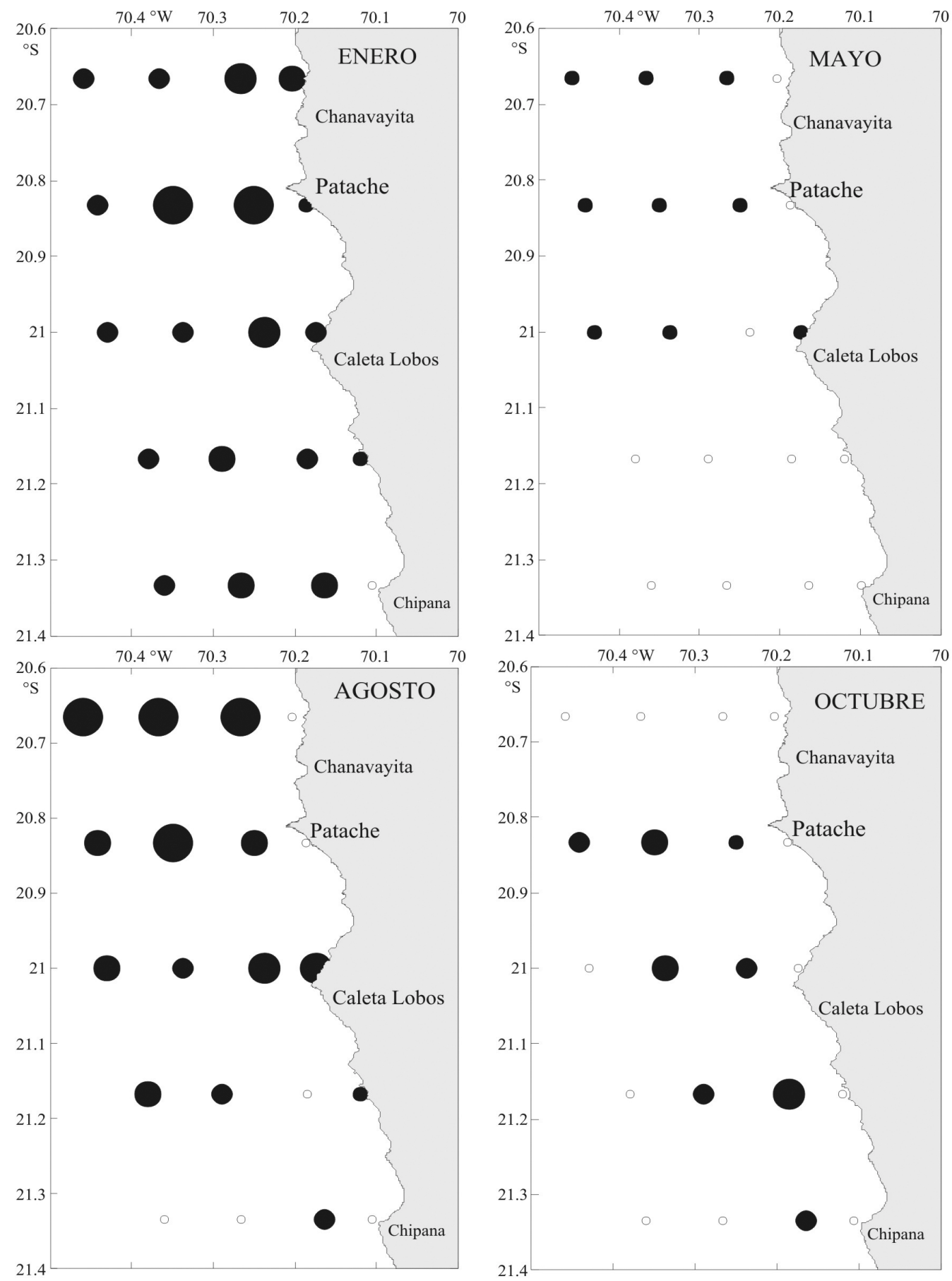

$0 \bigcirc 1$ a $64 \bigcirc 64$ a 365

365 a 1.400

1.400 a 4.200

4.200 a 11.500

Figura 4. Distribución temporal de la zoea I de langostino colorado en el norte de Chile (ind. $10 \mathbf{~ m}^{-2}$ ). Figure 4. Temporal distribution of the zoea I stage of the red squat lobster larvae from northern Chile (ind. $\cdot 10 \mathrm{~m}^{-2}$ ). 

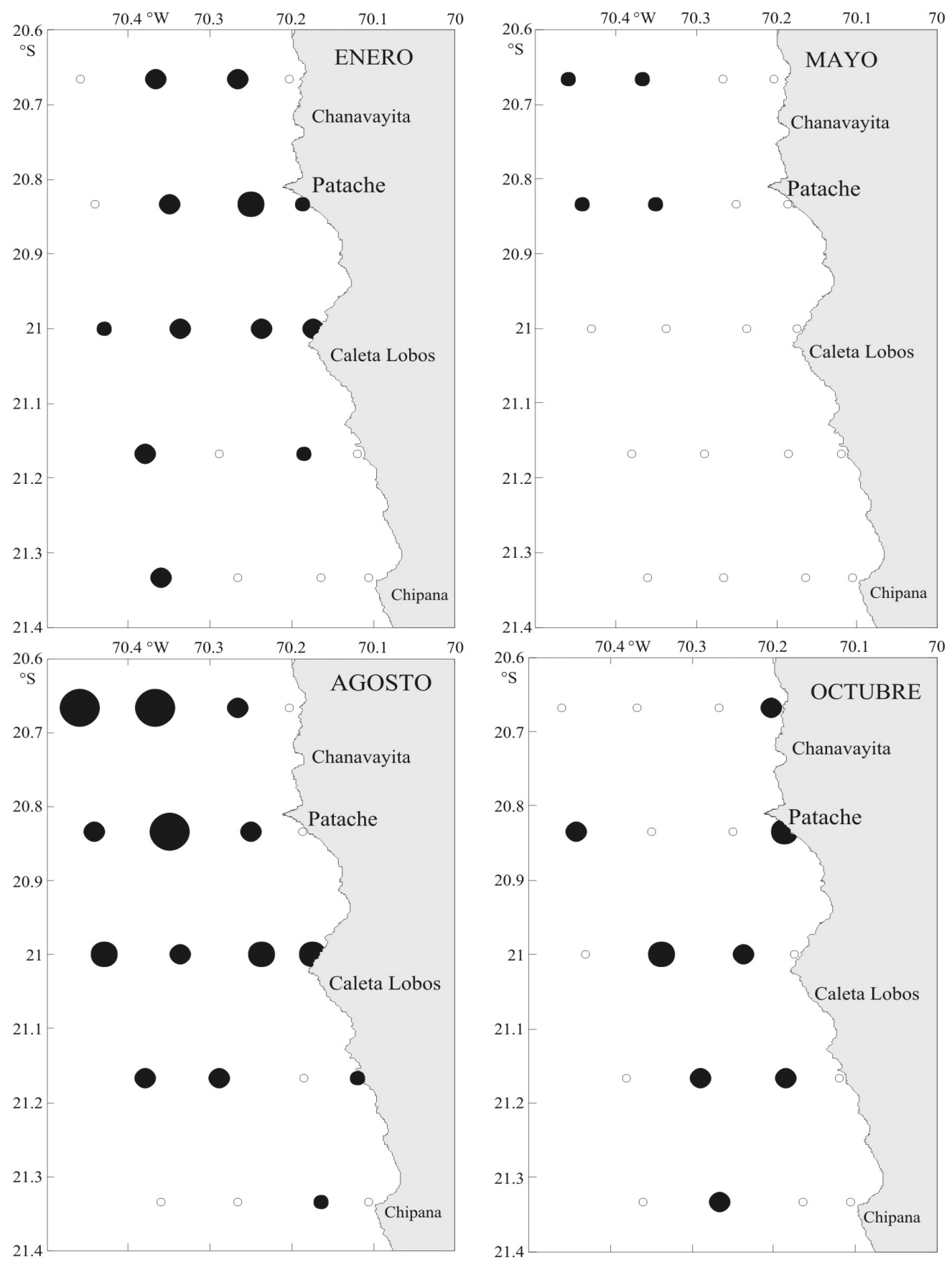

$0 \quad 1$ a $64 \bigcirc 64$ a 365

365 a 1.400

1.400 a 4.200

4.200 a 11.500

Figura 5. Distribución temporal de la zoea II de langostino colorado en el norte de Chile (ind. $\cdot 10 \mathbf{~ m}^{-2}$ ). Figure 5. Temporal distribution of the zoea II stage of the red squat lobster larvae from northern Chile (ind. $\cdot 10 \mathbf{~ m}^{-2}$ ). 

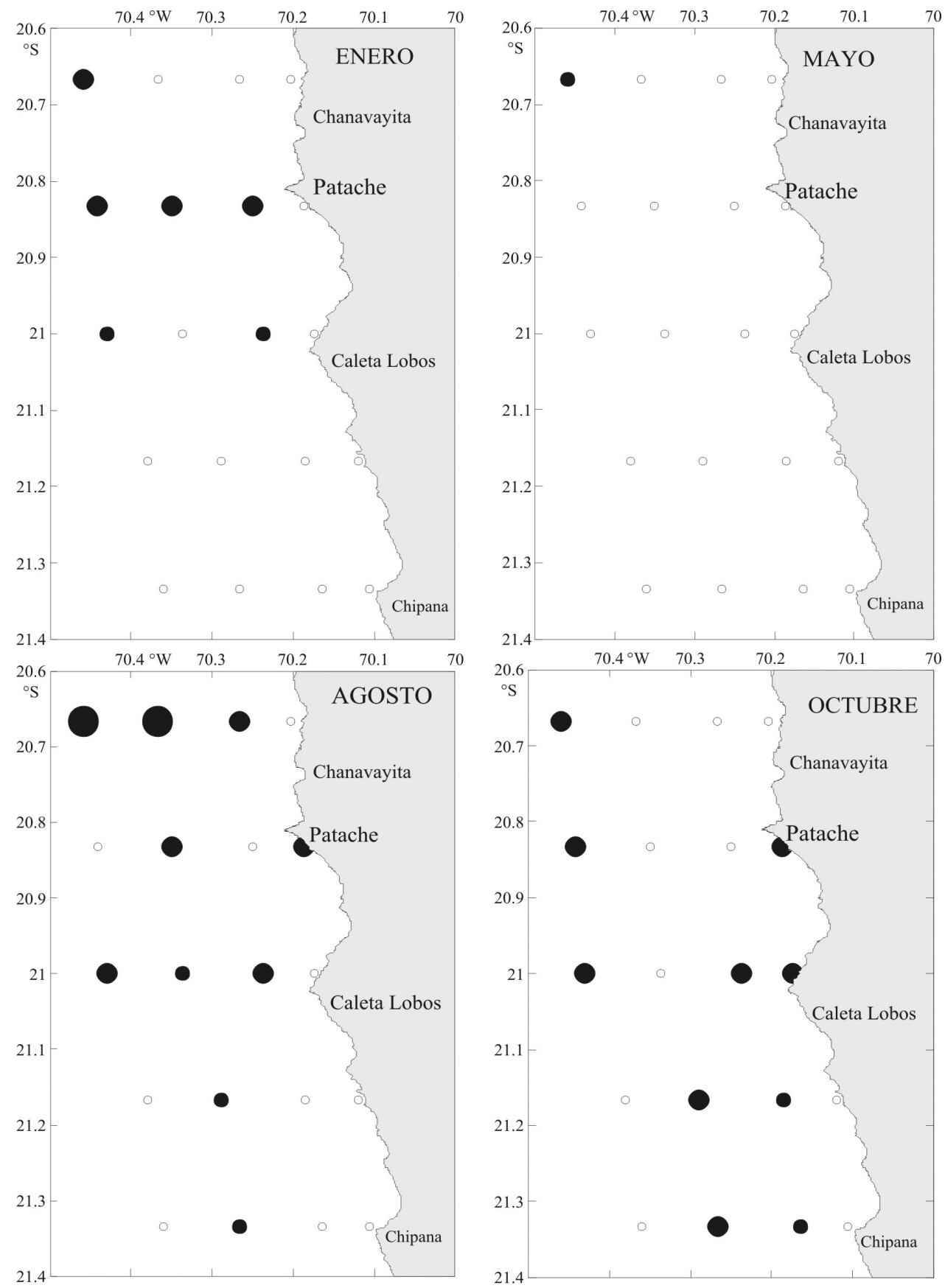

$0 \quad \bullet 1$ a 64

64 a 365

365 a 1.400

1.400 a 4.200

Figura 6. Distribución temporal de la zoea III de langostino colorado en el norte de Chile (ind. $\cdot \mathbf{1 0} \mathbf{~ m}^{-2}$ ). Figure 6. Temporal distribution of the zoea III stage of the red squat lobster larvae from northern Chile (ind. $\cdot 10 \mathbf{~ m}^{-2}$ ). 

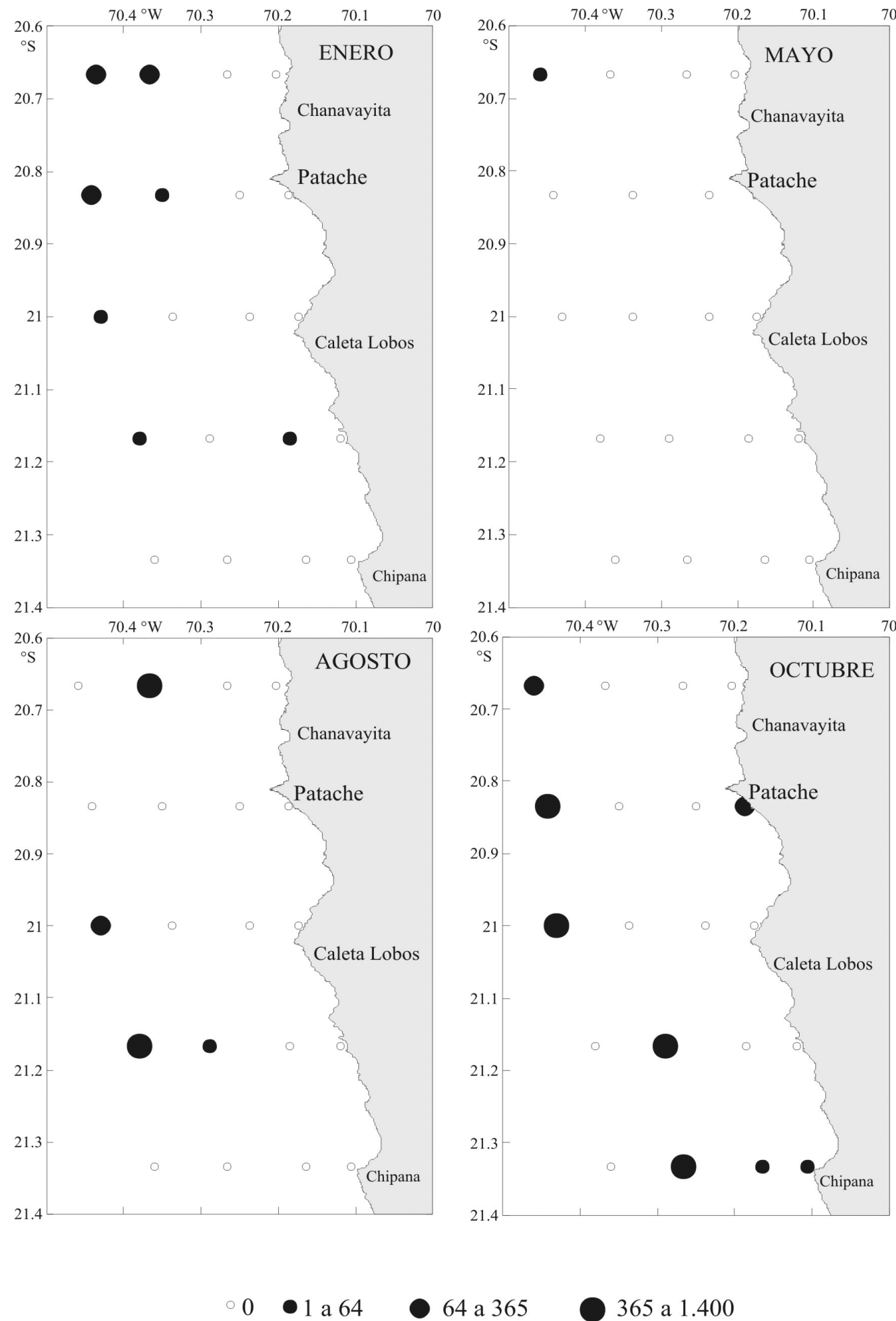

64 a 365

365 a 1.400

Figura 7. Distribución temporal de la zoea IV de langostino colorado en el norte de Chile (ind.・10 $\mathbf{m}^{-2}$ ). Figure 7. Temporal distribution of the zoea IV stage of the red squat lobster larvae from northern Chile (ind. $\cdot 10 \mathbf{~ m}^{-2}$ ). 

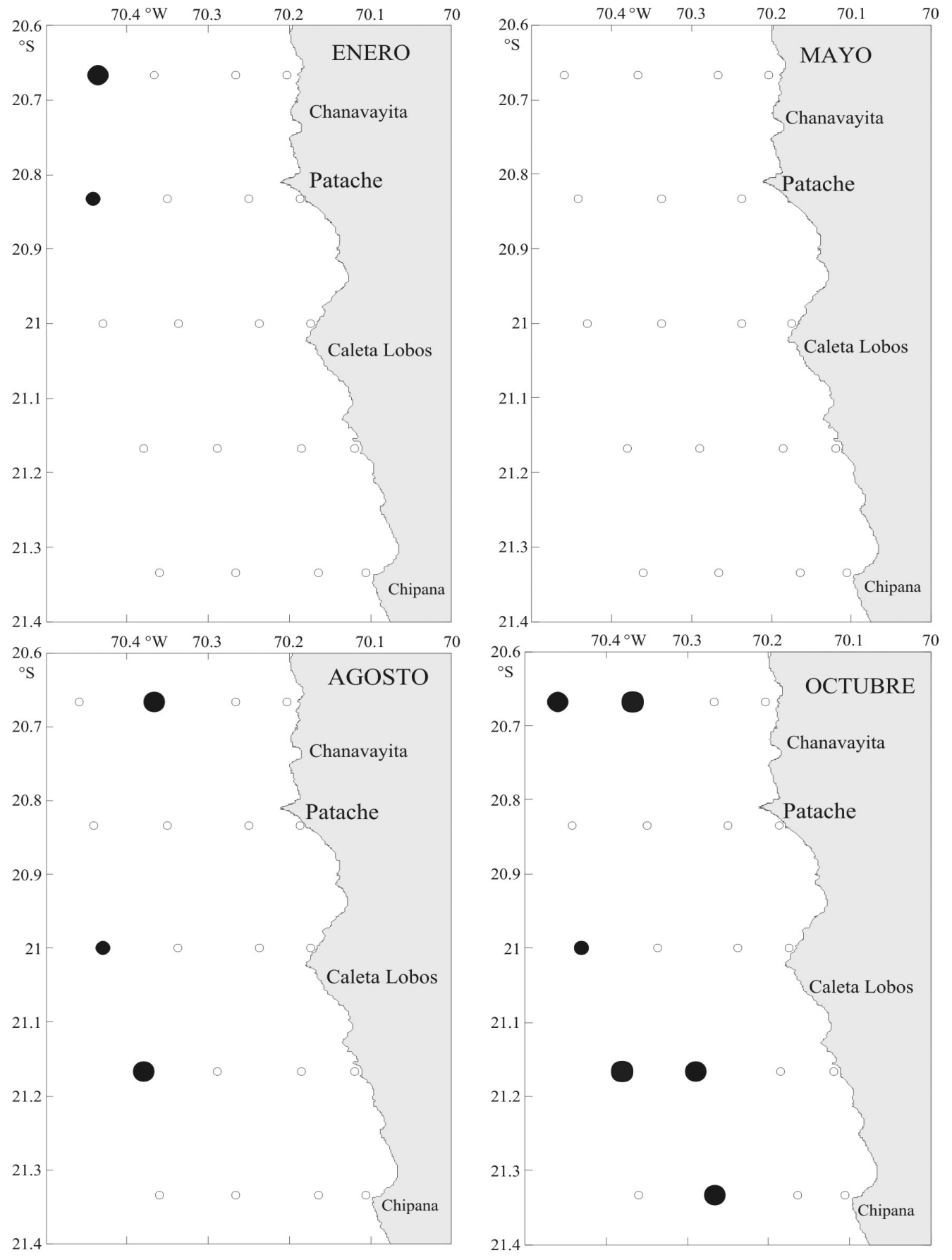

$0 \bigcirc 1$ a 64

64 a 365

Figura 8. Distribución temporal de la zoea $\mathrm{V}$ de langostino colorado en el norte de Chile (ind. $10 \mathbf{~ m}^{-2}$ ). Figure 8. Temporal distribution of the zoea $\mathrm{V}$ stage of the red squat lobster larvae from northern Chile (ind. $10 \mathrm{~m}^{-2}$ ). 

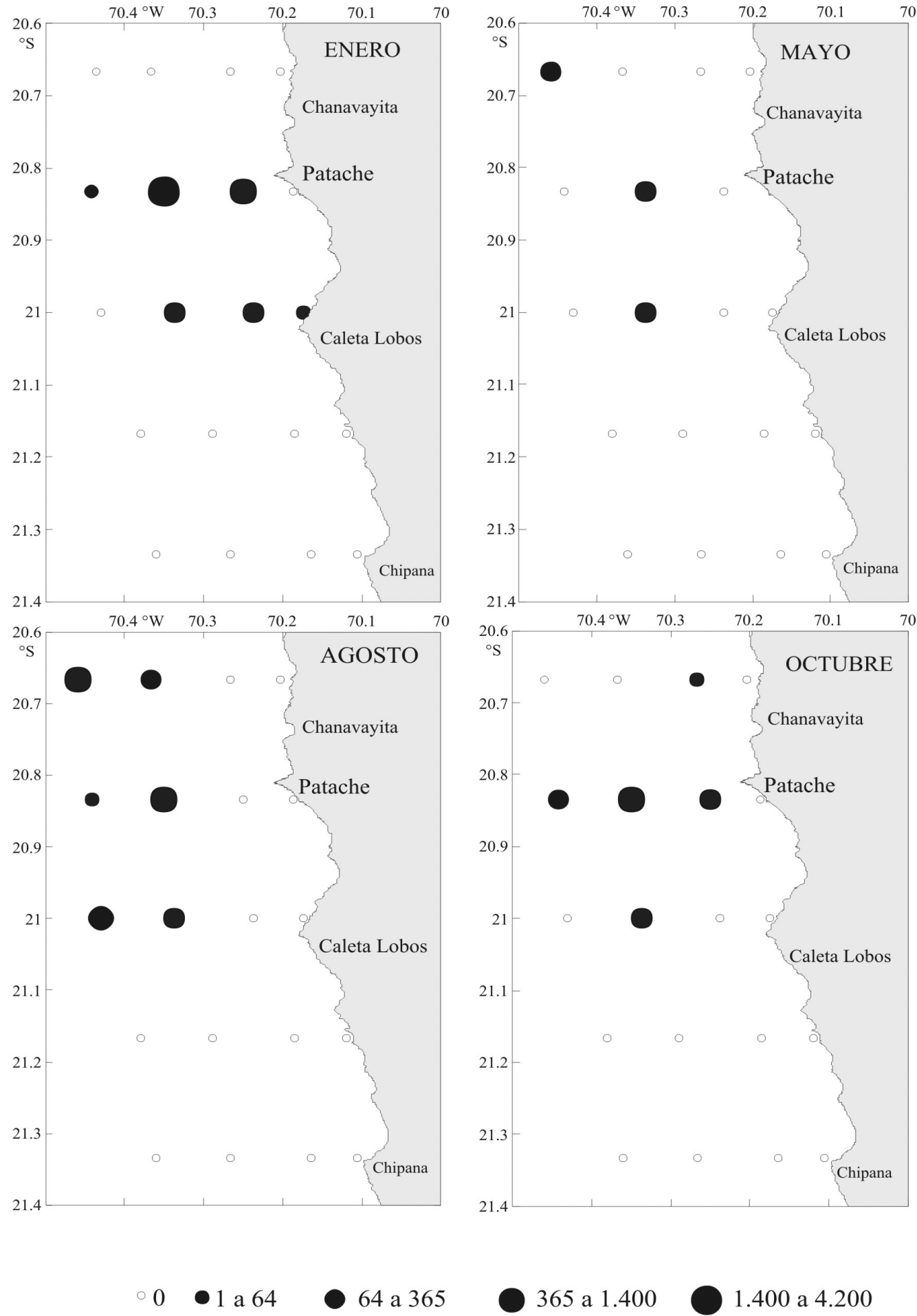

365 a 1.400

1.400 a 4.200

Figura 9. Distribución temporal de la megalopa de langostino colorado en el norte de Chile (ind. $\cdot 10 \mathbf{~ m}^{-2}$ ). Figure 9. Temporal distribution of the megalopa stage of the red squat lobster from northern Chile (ind. 10 $\mathbf{m}^{-2}$ ). 

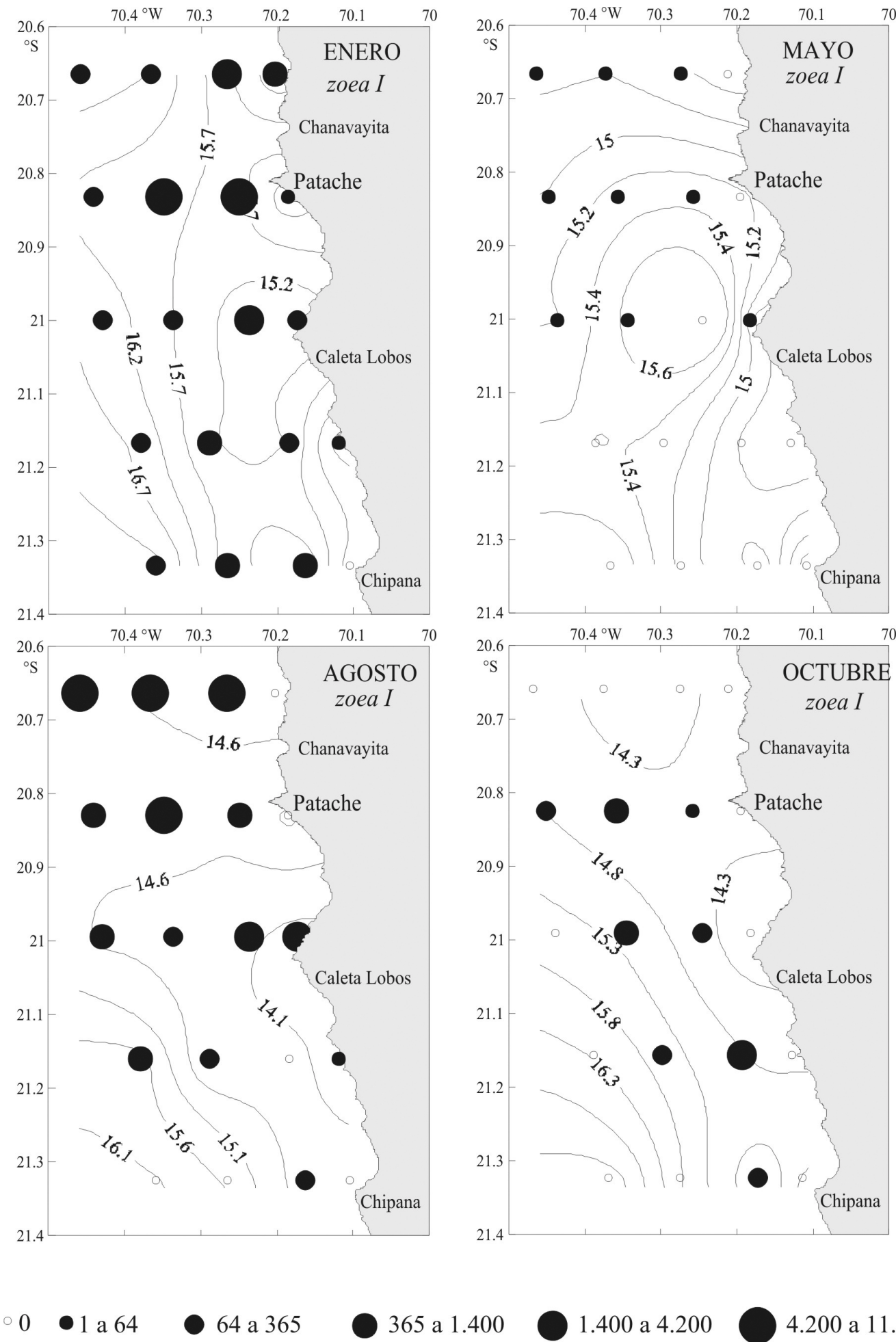

365 a 1.400

1.400 a 4.200

4.200 a 11.500

Figura 10. Relación temporal entre la zoea I de Pleuroncodes monodon y los contornos de los promedios ponderados de temperatura.

Figure 10. Temporal relationships between Pleuroncodes monodon zoea I stage larvae and temperature weighted mean contours. 

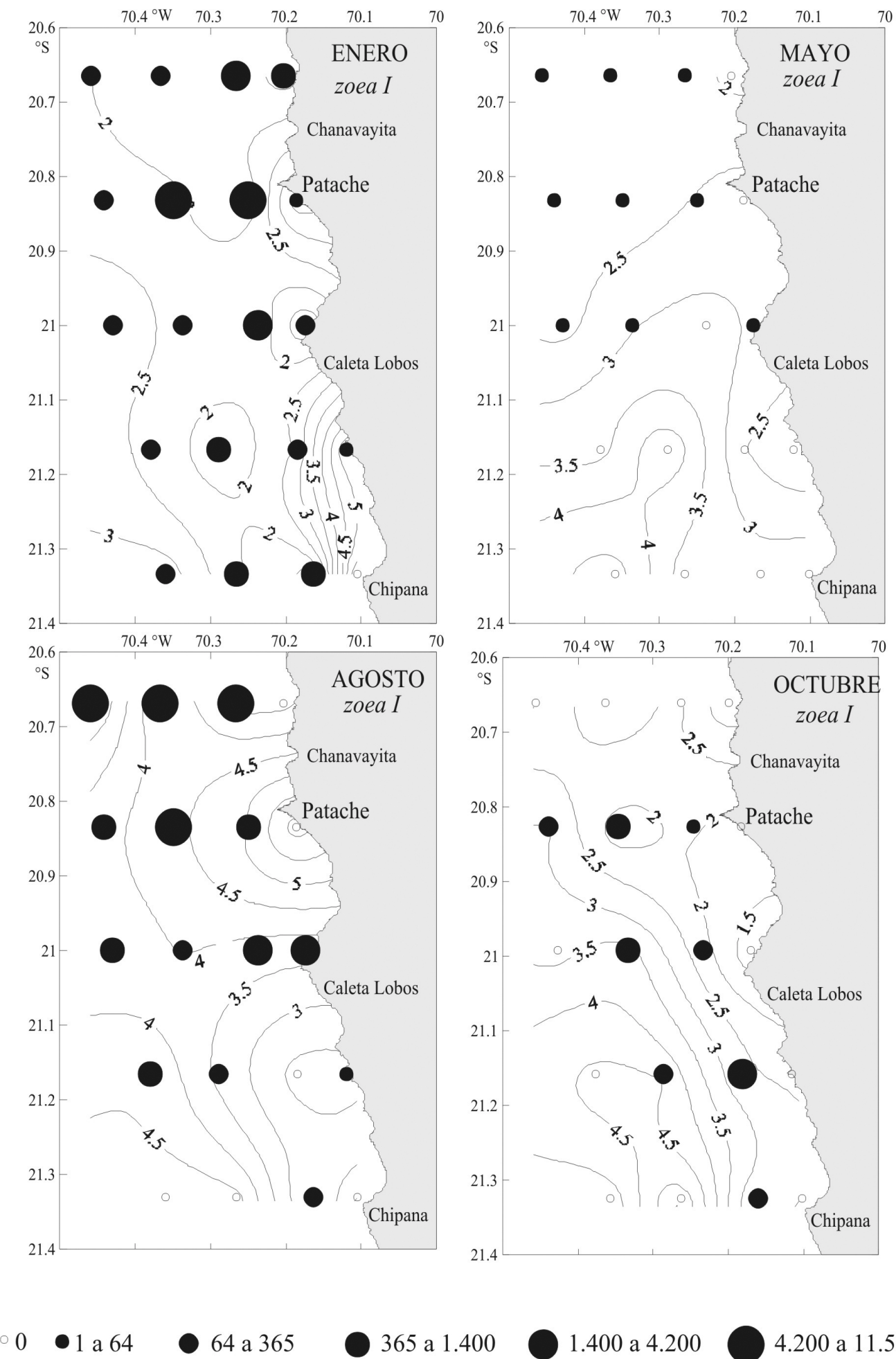

365 a 1.400

1.400 a 4.200

4.200 a 11.500

Figura 11. Relación temporal entre la zoea I de Pleuroncodes monodon y los contornos de los promedios ponderados de oxígeno disuelto.

Figure 11. Temporal relationships between Pleuroncodes monodon zoea I stage larvae and dissolved oxygen weighted mean contours. 

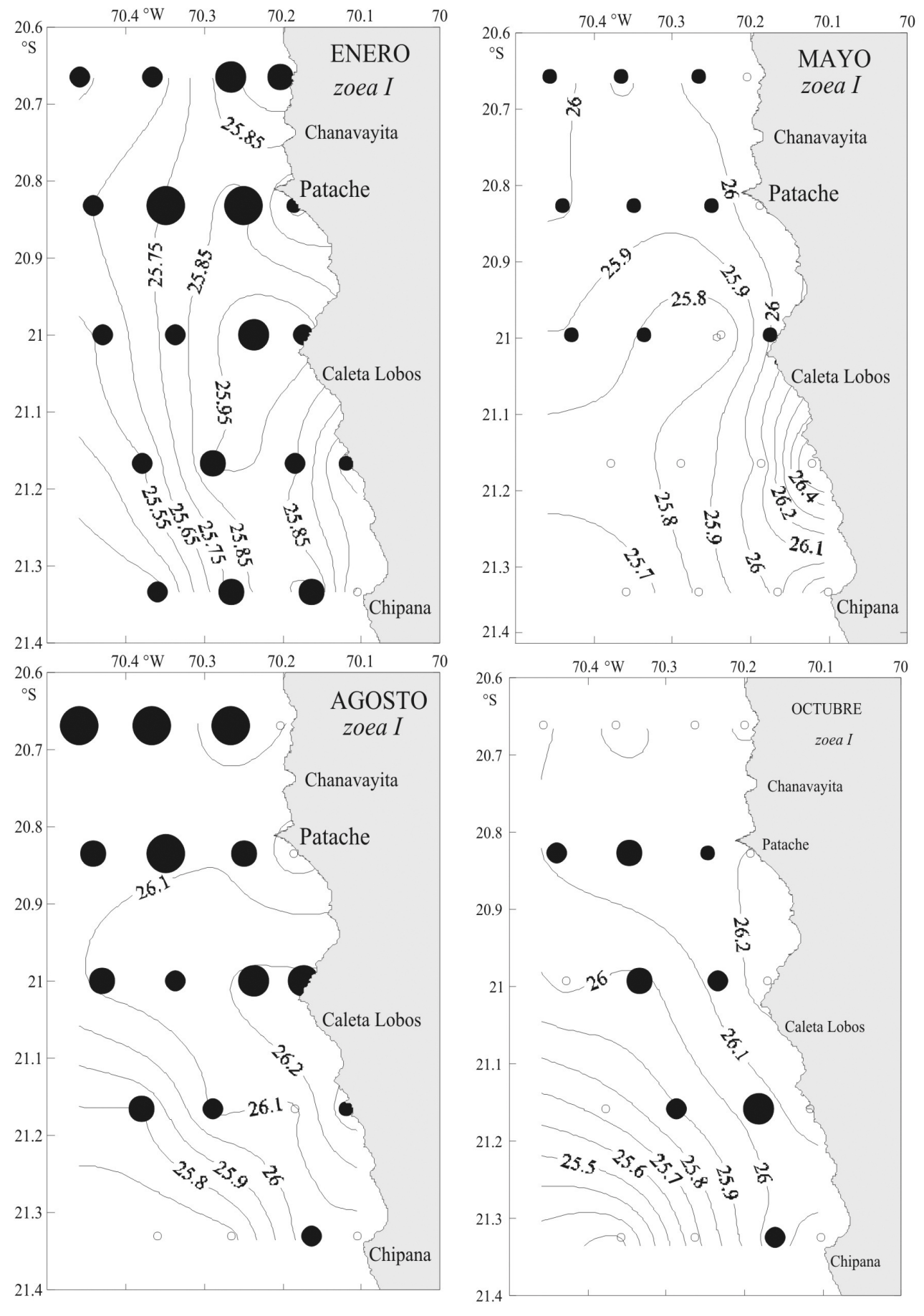

Figura 12. Relación temporal entre la zoea I de Pleuroncodes monodon y los contornos de los promedios ponderados de densidad.

Figure 12. Temporal relationships between Pleuroncodes monodon zoea I stage larvae and density weighted mean contours. 


\section{Asociación entre los distintos estadios larvales}

Se determinó el grado de asociación entre los diferentes estadios larvales y se encontró la presencia constante de dos grupos de estadios larvales en función del tiempo, los cuales tendrían relación con su dispersión y migración ontogénica.

En enero se observó que las zoeas III y IV presentaron una fuerte similitud (> 60\%) y se asociaron con la zoea $\mathrm{V}$, pero en menor grado. El otro grupo estuvo formado por las zoeas I, II y megalopa, pero con menor grado de similitud entre ellos (Fig. 13a). En mayo se determinó el mismo patrón entre las zoeas III y IV, separándose claramente del resto de los estadios, los cuales conformaron un grupo aparte (Fig. 13b). En agosto también se estableció la formación de dos grupos principales, aunque fueron diferentes a los meses anteriores, observándose un alto grado de asociación entre las zoeas I y II (> $80 \%$ ), que se separaron del resto de los estadios larvales, que presentaron una menor asociación entre ellos (Fig. 13c). En octubre se encontró una asociación entre zoea I y megalopa. No obstante, se destacó una baja similitud entre los diferentes estadios, lo que tendría relación con la amplia distribución de cada uno de ellos en el área de estudio en este periodo (Fig. 13d).

\section{DISCUSIÓN}

La distribución de organismos planctónicos como las larvas de $P$. monodon, se ve afectada principalmente por la dispersión causada por las corrientes marinas y la advección. Este proceso se inicia a partir del momento y lugar en que se efectúa la eclosión (áreas de eclosión) y finaliza con el reclutamiento a las poblaciones adultas (Boyd, 1967; Longhurst, 1968; Palma, 1994; Gómez \& Sánchez, 1997). En este sentido, para explicar las variaciones en la distribución y abundancia de los estadios larvales, es necesario determinar primero la temporada de reproducción, área de eclosión y factores ambientales que influyen en estas etapas de vida.

\section{Época reproductiva y tiempo de vida planctónica}

La reproducción de $P$. monodon a lo largo de la costa de Chile tiene una marcada estacionalidad, ajustada a la oceanografía regional de cada localidad.
En agosto se registraron las mayores abundancias larvales, siendo las zoeas I y II predominantes. Esto sugiere que dicho periodo correspondería al de mayor actividad reproductiva. Sin embargo, en enero también se observó una dominancia de zoea I, lo que sugiere un segundo periodo reproductivo, pero de menor magnitud. En agosto, la actividad reproductiva estaría adelantada con respecto a lo reportado por Mujica \& Bahamonde (1990) y Palma (1994) para la costa de Concepción, quienes señalan las mayores densidades larvales en primavera y las menores en otoño e invierno.

Las menores concentraciones de zoeas I y II se registraron en mayo, de manera similar a lo registrado en este mismo mes frente a Valparaíso y Concepción (Palma, 1976, 1980, 1994), lo cual se relacionaría con la relajación reproductiva que presenta esta especie tanto en la zona centro-sur, como en la zona norte del país en esa época del año.

Frente a la costa de Concepción, el langostino colorado presenta huevos durante casi seis meses, entre comienzos de mayo y fines de octubre, con una mayor incidencia de hembras ovíferas en agosto, estimándose un periodo de 90 a 120 días de desarrollo embrionario antes de la eclosión (Palma \& Arana, 1997). Sin embargo, las fluctuaciones estacionales de abundancia detectadas en el presente trabajo, se ajustarían más al ciclo reproductivo descrito para la bahía de Mejillones (Gutiérrez \& Zúñiga, 1977). Estos autores registraron hembras ovíferas durante todo el año, con la mayor incidencia (100\%) durante junio y julio, concordando con las altas densidades de zoea I registradas en agosto en el presente estudio.

La presencia de zoea I y megalopas en todas las estaciones del año, indica que la especie libera larvas y recluta individuos durante todo el año. No obstante, los antecedentes sobre el tiempo de vida planctónica de esta especie son variados. La duración media de los estadios zoea I a IV, indicaría que la vida larvaria en condiciones de laboratorio tendría una duración de al menos dos meses (Fagetti \& Campodonico, 1971) (Tabla 1). Sería razonable suponer que las mayores densidades de zoea IV registradas en octubre, se originaron en agosto, mientras que las megalopas que fueron dominantes en mayo, derivarían de eclosiones ocurridas en enero, comprendiendo un periodo de 3 a 4 meses de vida planctónica. Estas estimaciones coinciden en cierta medida, con los reportes de Gómez \& Sánchez $(1995,1997)$ para las larvas de $P$. planipes, que es- 

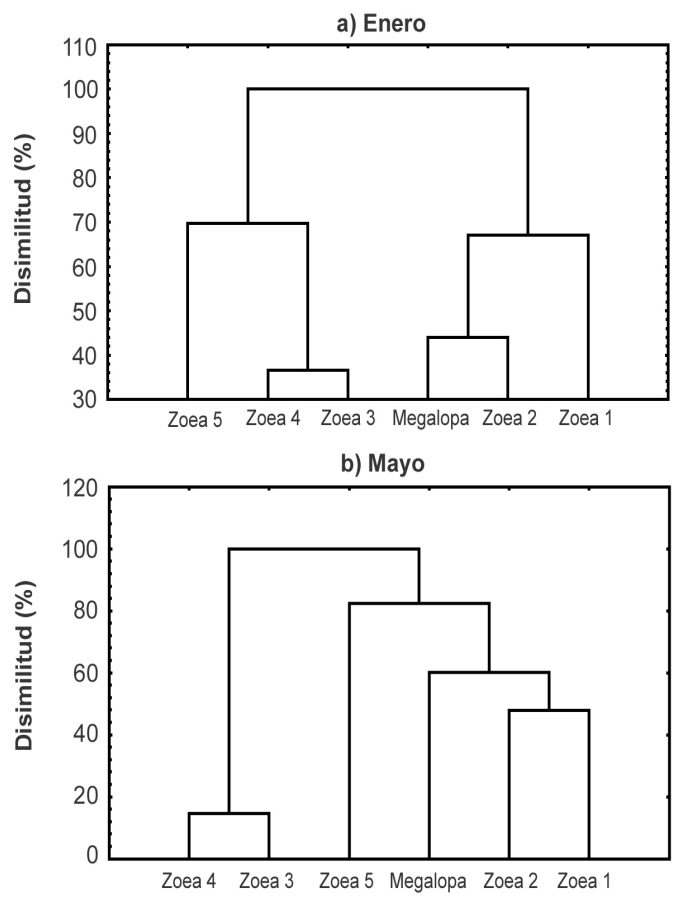

c) Agosto

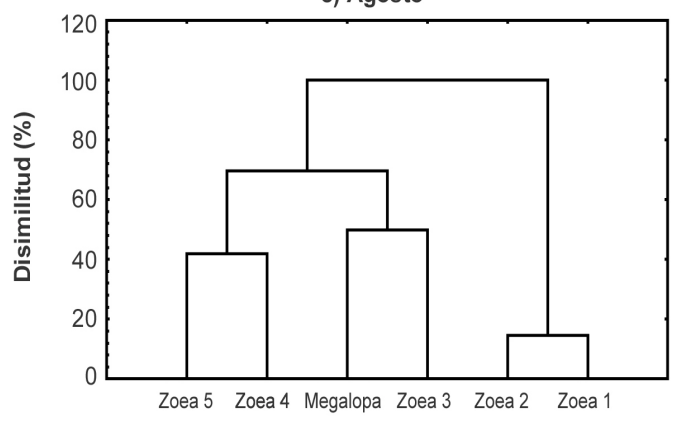

d) Octubre

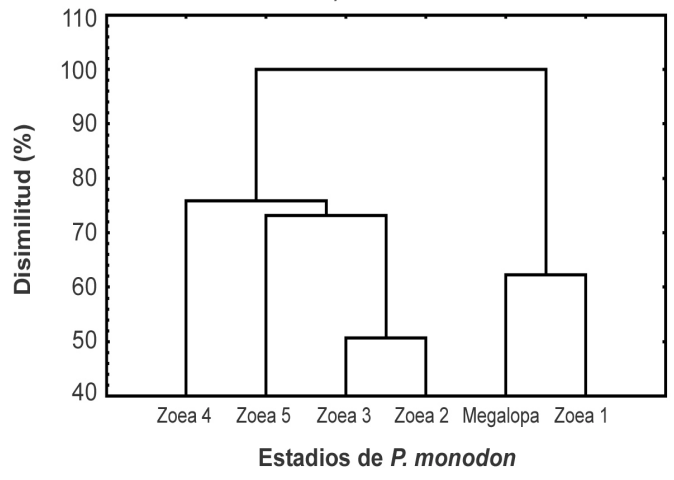

Figura 13. Dendrogramas de disimilitud entre los diferentes estadios larvales de langostino colorado en el norte de Chile, en cuatro estaciones del año.

Figure 13. Dissimilitude clusters between the different larval stages of the northern red squat lobster from northern Chile during the four seasons of the year. timaron un periodo de al menos cuatro meses hasta megalopa. No obstante, Gallardo et al. (1994) estimaron un lapso de vida larvaria de al menos cinco meses para $P$. monodon en bahía de Concepción, desde la eclosión primaveral en octubre-noviembre, hasta el reclutamiento a fines del verano y comienzos de otoño, aunque dicho periodo no coincide con la duración larvaria obtenida por Fagetti \& Campodonico (1971) en condiciones de laboratorio.

Los antecedentes descritos para su congénere del hemisferio norte, $P$. planipes (Stimpson), indican dos etapas en el ciclo reproductivo, con su máxima actividad en el periodo frío de enero y febrero (invierno del hemisferio norte), mientras que aquéllas del periodo cálido de julio y agosto (verano del hemisferio norte) constituirían reproducciones esporádicas o remanentes (Boyd, 1960; Longhurst, 1968; Gómez-Gutiérrez \& Sánchez-Ortiz, 1995, 1997). Estos antecedentes coinciden con los resultados obtenidos en este trabajo, en que el periodo reproductivo ocurriría principalmente en invierno (agosto), con producción larval esporádica o remanente en verano y otoño.

\section{Áreas de reproducción y variables ambientales}

El criterio utilizado para localizar áreas de eclosión fue la presencia de estadios tempranos de zoea I, debido a que éstos han estado expuestos a un periodo corto de deriva larval que varía entre 8 a 17 días (Fagetti \& Campodonico, 1971; Gómez-Gutiérrez \& Sánchez-Ortiz, 1995).

La abundancia de zoea I en relación con los promedios ponderados de las variables ambientales consideradas, no reflejaron relaciones significativas en ningún periodo analizado $(\mathrm{p}>0,05)$. Sin embargo, la distribución y abundancia de estas larvas evidenciaron dos periodos de actividad reproductiva, que se ajustan a las condiciones oceanográficas y a los eventos de surgencia que prevalecen en el área de estudio. Fuenzalida (1992) describió los procesos de surgencia para la misma zona $\left(20^{\circ} 30^{\prime}\right.$ $\left.21^{\circ} 45^{\prime} \mathrm{S}\right)$, detectando que estos prevalecen durante todo el año, indicando una señal anual con máximos de surgencia entre verano-otoño, declinando hasta alcanzar su mínima a comienzos de invierno, para luego volver a incrementar en el periodo de transición invierno-primavera con un segundo máximo anual. 
Tabla 1. Meses probables de eclosión larval de langostino colorado en el norte de Chile, de acuerdo al retrocálculo de los tiempos promedio de cada estadio de Pleuroncodes monodon.

Table 1. Probable months of larval appearance of red squat lobster off northern Chile, based on the retrocalculation of the time averages for each larval stage of Pleuroncodes monodon.

\begin{tabular}{|ccccc|}
\hline Estadio & $\begin{array}{c}\text { Duración } \\
\text { promedio (días) }\end{array}$ & $\begin{array}{c}\text { Tiempo } \\
\text { acumulado (días) }\end{array}$ & Referencia & $\begin{array}{c}\text { Meses probables } \\
\text { de eclosión }\end{array}$ \\
\hline zoea I & $10,3 \pm 4,9$ & 10,3 & Fagetti \& Campodonico (1971) & octubre \\
zoea II & $7,0 \pm 4,2$ & 17,3 & Fagetti \& Campodonico (1971) & septiembre-octubre \\
zoea III & $7,5 \pm 4,9$ & 24,8 & Fagetti \& Campodonico (1971) & septiembre \\
zoea IV & $29,9 \pm 19,8$ & 48,2 & Fagetti \& Campodonico (1971) & agosto \\
zoea V & $13,7 \pm 6,6$ & 54,7 & Boyd \& Johnson (1963) & agosto \\
megalopa & $?$ & $?$ & & julio-agosto? \\
\hline
\end{tabular}

\section{Áreas de eclosión y variables ambientales}

En la actividad reproductiva observada en agosto y en menor grado en enero de 2003, se observó una clara asociación con los eventos de surgencia que ocurren durante esos periodos en esta zona (Fuenzalida, 1992). En el presente estudio se observó una distribución norte-sur de la abundancia larvaria relacionada con la estructura física del área. Los focos de mayor abundancia de zoea I se asociaron con temperaturas promedios frías de $14,3^{\circ} \mathrm{C} \mathrm{y}$ $16,0^{\circ} \mathrm{C}$ en agosto y enero, respectivamente, valores que se registraron en las secciones norte y central principalmente (Fig. 10).

Las bajas densidades de zoea I registradas en el sector sur serían causadas por la presencia de frentes oceánicos (Fig. 10), debido a una condición dinámica constante que ocurre en el sector de punta Chipana y río Loa, donde se produce la intrusión de aguas más templadas hacia la costa, generándose frentes térmicos y de densidad en esos sectores (Fuenzalida, 1992), que restringen o inhiben parcialmente los pulsos de surgencia.

La asociación de los estadios tempranos a núcleos fríos de aguas costeras, también ha sido reportada para P. planipes en Baja California, evidenciando una asociación entre los centros de eclosión y los núcleos costeros de baja temperatura. Esto sugiere una estrategia reproductiva que favorece una mayor sobrevivencia larval (Longhurst, 1966; Gómez-Gutiérrez \& Sánchez-Ortiz, 1995, 1997), proceso que coincide con experimentos realizados en laboratorio (Fagetti \& Campodonico, 1971), donde se ha obtenido una mayor sobrevivencia larval de $P$. monodon a temperaturas de $15^{\circ} \mathrm{C}$, de manera similar a lo registrado en $P$. planipes (Boyd \& Johnson, 1963) y Galathea rostrata (Gore, 1979).

Si bien, la distribución del oxígeno disuelto fue relativamente concordante con la estructura térmica, los principales focos de zoea I se asociaron tanto a aguas bien oxigenadas $\left(4,53 \mathrm{~mL} \cdot \mathrm{L}^{-1}\right)$ en agosto, como a aguas pobres en oxígeno disuelto (1,25-2,96 $\left.\mathrm{mL} \cdot \mathrm{L}^{-1}\right)$ en enero. En cambio, en octubre los focos de abundancia se desplazaron hacia las secciones central y sur, con promedios más altos de oxígeno $\left(3,0 \mathrm{~mL} \cdot \mathrm{L}^{-1}\right)$ (Fig. 11), lo cual sugeriría un amplio rango de tolerancia larval con respecto a esta variable.

Se ha descrito la densidad como un buen indicador de los procesos de surgencia en la zona norte del país (Fuenzalida, 1992), siendo esta variable la que mejor se asoció a la distribución y dispersión de zoea I en el área de estudio. En enero, la abundancia de zoea I tuvo una distribución más homogénea, pero con una mayor concentración en el sector norte del área de estudio (Fig. 12). La amplia dispersión ocurrida en este mes, se explicaría por las bajas densidades observadas ( $25 \mathrm{~m}$ ), junto con los máximos índices de surgencia que ocurren en verano en esa área (Fuenzalida, 1992). Esta situación parece propicia para una mayor dispersión de larvas de decápodos en general (McConaugha, 1992).

En agosto, la alta concentración de zoeas I al norte de la sección central, estuvo asociada a la mayor homogeneidad en el campo de densidad (25 $\mathrm{m})$, dependiente de movimientos verticales en esos 
sectores. Estos movimientos tendrían relación con el incremento del índice de surgencia entre el periodo de invierno y primavera señalado por Fuenzalida (1992) para esta zona, evidenciándose nuevamente la relación entre distribución de áreas de eclosión de langostino colorado con eventos de surgencia detectados entre punta Patache y Pabellón de Pica (Fig. 12).

Los eventos de surgencia generan procesos de advección y mayor transporte de agua (Ekman), retirando las larvas de los ambientes favorables y afectando directamente la dinámica poblacional de algunos organismos meroplanctónicos (Bakun \& Parrish, 1982; Bakun, 1985). Al parecer, estos procesos no serian desfavorables para las larvas del langostino colorado, existiendo evidencias para suponer que la dispersión ontogénica costa-océano de las larvas, sería más bien parte de una estrategia reproductiva que estaría ocurriendo poco antes de las máximas surgencias, facilitando la dispersión y cumpliendo de esta manera un rol importante en el reclutamiento de estas especies (Longhurst, 1968, Palma, 1994; Gómez-Gutiérrez \& Sánchez-Ortiz, 1997).

En mayo se observó un acercamiento de los frentes térmicos y de densidad hacia la costa, junto con la intrusión de aguas más cálidas en casi toda el área de estudio. Esto indicó la presencia de movimientos verticales de menor intensidad en este periodo, situación que tendría relación con la señal anual del índice de surgencia, que decrece en el periodo de transición otoño-invierno (Fuenzalida, 1992). Siendo esta condición de fuertes estratificaciones y gradientes, al parecer poco favorable para la liberación de larvas al medio, implicaría una insuficiente dispersión como para cumplir los requerimientos ontogénicos de las larvas, situación que también se ha observado en larvas de $P$. planipes en Baja California (Gómez-Gutiérrez \& Sánchez-Ortiz, 1997).

\section{¿Área de crianza? y variables ambientales}

Resulta evidente que la alta productividad microplanctónica de estas regiones, propicia una mayor disponibilidad de alimento tanto para los estadios larvales como adultos. Existen reportes que las poblaciones adultas de $P$. monodon y $P$. planipes se concentran en esas regiones, principalmente, con fines de alimentación (Longhurst et al., 1967; Longhurst, 1968; Gutiérrez \& Zúñiga, 1977; Segu- ra \& Castillo, 1996; Gómez-Gutiérrez \& SánchezOrtiz, 1997).

La hipótesis de las áreas de crianza, descritas como áreas donde los juveniles pasan los primeros meses o años de vida en un área distinta de la población adulta (Roa et al., 1995), se relacionaría con regiones con regímenes permanentes de eventos de surgencia, asociados a bajas concentraciones de oxígeno disuelto y alta disponibilidad de alimento (Arcos \& Wilson, 1984). Con respecto a esto, Roa et al. (1995) establecieron las áreas o sustratos de crianza para $P$. monodon frente a la costa de Concepción, donde el asentamiento de juveniles estaría relacionado con la relajación de los regímenes de surgencia, como con el desarrollo de las colonias de la bacteria Thioploca sp., detectándose una clara estacionalidad (Gallardo et al., 1994).

No obstante, Longhurst (1966) señaló que las zoeas de $P$. planipes pueden permanecer cerca de la costa durante su periodo larval para reclutar directamente al stock de subadultos pelágicos costeros o ser transportados mar afuera como postlarvas para asentarse posteriormente a mayor profundidad. Se hace necesario destacar, que la máxima actividad reproductiva del langostino colorado del norte, tanto la de agosto como la de enero, ocurren coincidentemente junto con los máximos índices de surgencia y transporte costa afuera, situación similar a la encontrada en P. planipes en Baja California (Gómez-Gutiérrez \& Sánchez-Ortiz, 1995, 1997).

En este trabajo se observó la presencia de megalopas durante los cuatro periodos de estudio, asociada a estadios tempranos, zoea I y II, en las secciones norte y central, no registrándose en el sector sur (Figs. 9 y 13). Esto, estaría evidenciando una permanencia de las megalopas en dicha zona, relacionada con su área de crianza, generando de esta manera suficientes reclutas para mantener la población pelágica, concordando en cierta manera con lo señalado por Longhurst (1966).

Estas zonas, sometidas a constantes regímenes de surgencia, con fuertes transportes costa afuera (Ekman), generarían las condiciones para la formación de celdas costeras de circulación, que podrían limitar con las corrientes preponderantes a lo largo de la costa (Roughgarden et al., 1985, 1988; McConaugha, 1992), como sería en este caso la corriente de Humboldt. Sin embargo, otros estudios han demostrado mecanismos de retorno y transporte de organismos meroplanctónicos hacia la costa, coincidentes con la relajación de los regímenes de 
surgencia y el posterior acercamiento de los frentes hacia zonas costeras (Wing et al., 1995, 1998; Shanks et al., 2000), más relacionado con lo observado en el presente estudio. Por esto, es necesario realizar estudios con una cobertura mayor del área oceánica que permitan detectar y dilucidar las interacciones físico-biológicas a mesoescala en el área de estudio.

\section{Consideraciones finales}

Se ha sugerido un clino latitudinal de la actividad reproductiva de $P$. planipes a lo largo de la costa de Baja California (Rodríguez et al., 1995; Gómez \& Sánchez, 1997), sobre la base de diferencias temporales en la madurez gonadal, así como en las tallas de los individuos adultos y de los estadios larvales a diferentes latitudes, encontrándose tallas menores en latitudes más bajas y templadas. Los antecedentes aquí expuestos, sumados a los existentes para las aguas del centro-sur de Chile, permitirían sugerir una situación similar que aquella para su congénere del hemisferio norte (S. Palma, com. pers.). Si bien, la presencia de clinos reproductivos latitudinales ya ha sido observada en otros crustáceos decápodos (Lardies \& Castilla, 2001), es preciso contar con mayor información de madurez gonadal, distribución espacio-temporal, así como de tallas de los estadios larvales de la zona norte y centro-sur de Chile, para poder comprender más profundamente el ciclo de vida de esta especie.

La característica más relevante del langostino colorado del norte es sin duda su hábito pelágico, diferente al de las poblaciones de la zona centrosur, que son de hábitos bentodemersales (Gallardo et al., 1993, 1994). No obstante, esta característica pelágica ha sido comúnmente reportada para $P$. planipes por Longhurst (1966, 1967), Boyd (1967) y Aureoles-Gamboa (1995) y en Munida gregaria por Zeldis (1985). Este alargamiento de la fase pelágica y del trasporte costa-océano de los estadios larvales no es desfavorable para la sobrevivencia de estas especies (Gómez-Gutiérrez \& SánchezOrtiz, 1997). En P. monodon, esta podría ser una adaptación a las características topográficas y oceanográficas en que habita, como son la estrecha plataforma continental y la presencia constante de la capa mínima de oxígeno en la zona norte (Fuenzalida, 1992; Morales et al., 1996).

Sin embargo, la separación de los diferentes estadios de vida (planctónicos, pelágicos y bentónicos) de estas especies, podría sugerir una estrategia para disminuir la competencia intraespecífica por el alimento, como por el canibalismo entre ellas (Zeldis, 1985; Gómez-Gutiérrez \& Sánchez-Ortiz, 1997). No obstante, este particular ciclo de vida, donde los diferentes estadios larvales permanecen espacialmente segregados, podría explicar como estas especies son capaces de desarrollar altas concentraciones en los sistemas de surgencia costera del norte de Chile.

\section{AGRADECIMIENTOS}

Se desea expresar nuestros más sinceros agradecimientos al Programa de Oceanografía Pesquera (PROPNOR) y a Corpesca S.A., por la realización de estos cruceros de investigación marina en el norte de Chile. En especial al coordinador de dicho programa Sr. Gabriel Claramunt Q., quien ha facilitado las muestras y todo su apoyo para este trabajo. De igual manera a los ayudantes del laboratorio de Biología Pesquera de la Universidad Arturo Prat, Samuel Soto y Cristián Azócar por su buena disposición durante el desarrollo de este trabajo. A nuestro amigo y colega Guillermo Guzmán por sus comentarios críticos y literatura sobre $P$. monodon. Finalmente, agradecemos los comentarios y sugerencias de los revisores anónimos.

\section{REFERENCIAS}

Arcos D.F. \& R.E. Wilson. 1984. Upwelling and the distribution of chlorophyll $a$ within the Bay of Concepción, Chile. Estuar. Coast. Shelf. Sci., 92: 254-268.

Aurioles-Gamboa, D. 1995. Migración batimétrica de la langostilla bentónica en la plataforma continental del Pacífico de Baja California. En: D. AuriolesGamboa \& E. Balart (eds.). La langostilla: biología, ecología y aprovechamiento. Centro de Investigaciones Biológicas del Noroeste, S.C., pp. 79 92.

Bakun, A. 1985. Comparative studies and the recruitment problem searching for generalization. Calif. Coop. Ocean. Fish. Invest., 19: 107-127.

Bakun, A. \& R.H. Parrish. 1982. Turbulence transport and fish in California and Perú Current. Calif. Coop. Ocean. Fish. Invest., 22: 99-111.

Boyd, C. 1960. The larval stages of Pleuroncodes planipes Stimpson (Crustacea, Decapoda, 
Galatheidae). Biol. Bull. Mar. Biol. Lab. Woods Hole, 118: 17-30.

Boyd, C. 1967. Benthic and pelagic habitats of the red crabs Pleuroncodes planipes. Pac. Sci., 21: 394403.

Boyd, C. \& M. Johnson. 1963. Variations in the larval stages of decapod crustacean, Pleuroncodes planipes Stimpson (Galatheidae). Bull. Scripps Inst. Oceanogr., 124: 249-251.

Brower, J., J. Zar \& C. von Ende. 1997. Field and laboratory methods for general ecology. McGrawHill, Boston, 273 pp.

Bustos, H., O. Aracena, S. Mora \& W. Palma. 1982. Estudio de crecimiento y edad en el recurso langostino colorado (Pleuroncodes monodon H. Milne Edwards, 1837). Inf. Inst. Pesq., Santiago, 120 pp.

Christiansen, M. \& K. Anger. 1990. Complete larval development of Galathea intermedia Lilljeborg Reared in laboratory Culture (Anomura: Galatheidae). J. Crust. Biol., 10(1): 87-111.

Fagetti, E. \& I. Campodonico. 1971. Larval development of the red crab Pleuroncodes monodon (Decapoda, Anomura, Galatheidae) under laboratory conditions. Mar. Biol., 8(1): 70-81.

Frontier, S. 1981. Tratamiento de los datos. En: D. Boltovskoy (ed.). Atlas de zooplancton del Atlántico sudoccidental y métodos de trabajo con el zooplancton marino. Inst. Nac. Invest. Des. Pesq. (INIDEP), Mar del Plata, pp. 169-188.

Fuenzalida, R. 1992. Procesos de surgencia en la región norte de Chile, latitudes $20^{\circ} 30^{\prime} \mathrm{S}-21^{\circ} 45^{\prime} \mathrm{S}$. Invest. Cient. Tec., Ser. Ciencias del Mar, 2: 1230 .

Gallardo, V., I. Cañete, S. Enríquez-Brionez, R. Roa, A. Acuña \& M. Baltazar. 1993. Biología del langostino colorado Pleuroncodes monodon $\mathrm{H}$. Milne Edwards, 1837 y especies afines (Crustacea, Decapoda, Anomura, Galatheidae): sinopsis. En: F. Faranda \& O. Parra (eds.). Elementos básicos para la gestión de los recursos vivos marinos costeros de la región del Biobío. Programa EULA, Universidad de Concepción, Monografías Científicas, 2: 67-113.

Gallardo, V., I. Cañete, R. Roa, S. Enríquez-Brionez \& M. Baltazar. 1994. Recruitment of the squat lobster Pleuroncodes monodon on the continental shelf off central Chile. J. Crust. Biol., 14(4): 665-669.
Gómez-Gutiérrez, J. \& C. Sánchez-Ortiz. 1995. Centros de eclosión y deriva larval y postlarval de la langostilla, Pleuroncodes planipes (Crustacea: Galatheidae), en la costa occidental de Baja California sur. En: D. Aurioles-Gamboa \& E. Balart (eds.). La langostilla: biología, ecología y aprovechamiento. Centro de Investigaciones Biológicas del Noroeste, S.C., pp. 35-57.

Gómez-Gutiérrez, J. \& C. Sánchez-Ortiz. 1997. Larval drift and population structure of the pelagic phase of Pleuroncodes planipes (Stimpson) (Crustacea: Galatheidae) off the southwest coast of Baja California, Mexico. Bull. Mar. Sci., 61(2): 305-325.

Gore, R. 1979. Larval development of Galathea rostrata under laboratory conditions, with a discussion of larval development in the Galatheidae (Crustacea Anomura). U.S. Fish. Bull., 76(4): 781806.

Gutiérrez, J. \& O. Zúñiga. 1977. Pleuroncodes monodon H. Milne Edwards, 1837 en la bahía de Mejillones del sur, Chile (Crustacea, Decapoda, Anomura). Rev. Biol. Mar, Valparaíso, 16(2): 161169.

Haig, J. 1955. The crustacean Anomura of Chile. Report of the Lunds University Chile Expedition 1848-49. Lund Univ. Arsskr. (N.F. Avd. 2), 51(12): $1-60$.

Lardies, M. \& J.C. Castilla. 2001. Latitudinal variation in the reproductive biology of the commensal crab Pinnaxodes chilensis (Decapoda: Pinnotheridae) along the Chilean coast. Mar. Biol., 139: 1125-1133.

Longhurst, A. 1966. The pelagic phase of Pleuroncodes planipes in the California Current. Calif. Coop. Ocean. Fish. Invest., 11: 142-154.

Longhurst, A. 1968. Distribution of the larval stages of the Pleuroncodes planipes in the California Current. Limnol. Oceanogr., 13(1): 143-155.

Longhurst, A., C.J. Lorenzen \& W.H. Thomas. 1967. The role of pelagic crabs in the grazing of phytoplankton off Baja California. Ecology, 48: 190-200.

McConaugha, J. 1992. Decapod larvae: dispersal, mortality, and ecology. A working hypothesis. Amer. Zool., 32: 512-523.

Morales C., M. Braun, H. Reyes, J. Blanco \& A. 
Davies. 1996. Anchovy larval distribution in the coastal zone off northern Chile: the effect of low dissolved oxygen concentration and of a cold-warm sequence (1990-95). Invest. Mar., Valparaíso, 24: 77-96.

Mujica, A. \& N. Bahamonde. 1990. Larvas de Galatheidae en plancton marino del área de Talcahuano (Crustacea, Decapoda, Anomura). Manuscrito no publicado, $15 \mathrm{pp}$.

Palma, S. 1976. Meroplancton de la región de Valparaíso. Cienc. Tecnol. Mar, 2: 99-116.

Palma, S. 1980. Larvas de crustáceos decápodos capturadas frente a la costa de Valparaíso. Invest. Mar., Valparaíso, 8(1-2): 129-144.

Palma, S. 1994. Distribución y abundancia de larvas de langostino colorado Pleuroncodes monodon frente a la costa de Concepción, Chile. Invest. Mar., Valparaíso, 22: 13-29.

Palma, S. \& P. Arana. 1997. Aspectos reproductivos del langostino colorado (Pleuroncodes monodon H. Milne Edwards, 1837), frente a la costa de Concepción, Chile. Invest. Mar., Valparaíso, 27: 203221.

Rayner, G. 1935. The Falkland species of the crustacean genusMunida. Discovery Rep., 10: 209245.

Retamal, M.A. 1981. Catálogo ilustrado de los crustáceos decápodos de Chile. Gayana Zool., 44: 110.

Roa. R., V. Gallardo, B. Ernst, M. Baltazar, J. Canete \& S. Enríquez-Briones. 1995. Nursery ground, age structure and abundance of juvenile squat lobster Pleuroncodes monodon on the continental shelf off central Chile. Mar. Ecol. Progr. Ser., 116: 47-54.

Rodríguez, M., V. Serrano \& D. Aureoles-Gamboa. 1995. Biología reproductiva de la langostilla en la costa occidental de Baja California sur. En: D. Aurioles-Gamboa \& E. Balart (eds.). La langostilla: biología, ecología y aprovechamiento. Centro de Investigaciones Biológicas del Noroeste, S.C., pp. 93-107.

Recibido: 14 agosto 2004; Aceptado: 18 marzo 2005
Rosales, S. 1992. Estructura comunitaria horizontal de copépodos calanoídeos y características hidrográficas entre Los Vilos y Valparaíso, durante enero de 1990. Tesis de Oceanografía, Escuela de Ciencias del Mar, Universidad Católica de Valparaíso, Valparaíso, 85 pp.

Roughgarden, J., Y. Iwasa \& C. Baster. 1985. Demographic theory for an open marine population with space-limited recrutment. Ecology, 66: 54-67.

Roughgarden, J., S. Gaines \& H. Possingham. 1988. Recruitment dynamics in complex life cycles. Science, 241: 1460-1466.

Segura, M. \& R. Castillo. 1996. Distribución y concentración de la "Munida" (Pleuroncodes monodon) en el verano 1996. Inf. Inst. Mar Perú, 122: $79-85$.

Seridji, R. 1995. On a collection of decapod larvae from the gulf of Aqaba off the Jordian coast, I. Thalassinidea and Anomura. Crustaceana, 68: 281220.

Shanks, A., J. Largier \& L. Brink. 2000. Demonstration of the onshore transport of larval invertebrates by the shoreward movement of an upwelling front. Limnol. Oceanogr., 45(1): 230 236.

Ulloa, R. \& S. Palma. 1998. Distribución larval de Petrolisthes violaceus, $P$. laevigatus y Allopetrolisthes angulosus en el plancton de la bahía de Valparaíso (Crustacea, Anomura, Porcellanidae). Rev. Biol. Mar. Oceanogr., 33(1): 125-138.

Wing, S.R., L.W. Botsford, J. Largier \& L. Morgan. 1995. Spatial structure of relaxation events and crab settlement in the northern California Upwelling System. Mar. Ecol. Prog. Ser., 128: 199-211.

Wing, S., L. Botsford, S. Ralston \& J. Largier. 1998. Meroplanktonic distribution and circulation in a coastal retention zone of the northern California Upwelling System. Limnol. Oceanogr., 43(7): 1710-1721.

Zeldis, J.R. 1985. Ecology of Munida gregaria (Decapoda, Anomura): distribution and abundance, population dynamics and fisheries. Mar. Ecol. Progr. Ser., 22: 77-99. 